\title{
SÍNTESE HIDROTERMAL ASSISTIDA POR MICROONDAS DO ÓXIDO DE ZINCO DOPADO COM BISMUTO E SUA CARACTERIZAÇÃO
}

\author{
Camila Stockey Erhardt ${ }^{1}{ }^{*}$, Carlos Pérez Bergmann ${ }^{1}$, Adriane de Assis Lawisch Rodriguez ${ }^{2}$ \\ 1 Departamento de Materiais (PPGE3M), Escola de Engenharia, Universidade Federal do Rio Grande do Sul, 90035190, Rio Grande \\ do Sul, Brasil. \\ 2 Departamento de Engenharia, Arquitetura e Ciências Agrárias, PPGTA, Universidade de Santa Cruz do Sul, 96815-900, Rio \\ Grande do Sul, Brasil
}

*E-mail: camilaerhardt1@gmail.com

Recebido em: 10/01/2018

Aceito em: 10/02/2018

\section{RESUMO}

O óxido de zinco devido à presença de defeitos intrínsecos possibilita a dopagem com outros materiais, como o bismuto. O BZO (óxido de zinco dopado com bismuto) preparado via síntese hidrotermal assistida por microondas (MAHS) foi estudado por sua concentração de dopante e caracterização morfológica. Os produtos foram caracterizados DRX, MEV e PL; Cálculos de banda gap por espectroscopia de transmissão. Os resultados indicam que as nanoestruturas de BZO foram obtidas com tamanhos de cristalitos variando de 5,82 a 8,37 nm. A mesma estrutura morfológica, tipo flor, foi formada com diferentes concentrações de dopantes. PL demonstra que os defeitos aumentam com a dopagem do bismuto. $\mathrm{O}$ intervalo de band gap encontrado foi de 2,79-3,3 eV e tem potencial uso em aplicações de fotodegradação.

Palavras-chave: Síntese hidrotermal. Microondas. Óxido de zinco. Dopante. Bismuto.

\section{Introdução}

O óxido de zinco é um dos materiais mais eficientes e promissores no sistema de gestão ambiental, tem como aplicação a purificação da água, tratamento de água, processo de autolimpeza, sínteses orgânicas e reações de desinfecção. Já para a aplicação em energia, o óxido de zinco serve como redução de $\mathrm{CO}_{2}$ para combustíveis de hidrocarbonetos e energia renovável [12].

O óxido de zinco é reconhecido como uma material fotocatalítico que possui uma grande fotosensitividade [3], baixo custo, viabilidade e disponibilidade para produção. Considerado um semicondutor do grupo II-IV [4], com um amplo band gap $(3,37 \mathrm{eV})$ e grande energia de ligação $(\mathrm{Eg}=60 \mathrm{meV})[1,3-11]$.

A síntese e dopagem do óxido de zinco de zinco pode ser realizada por diversos métodos não somente o método hidrotermal assistido por microondas $[4,6,9,13]$, mas também por síntese eletroquímica[14], solvotermal [15], sonoquimica [16], deposição de vapor [17], co-preciptação [18], wet chemical [19], microemulsão[20] e sol-gel [21].

A síntese hidrotermal é considerada um dos métodos de síntese mais convenientes e práticos, pois não necessita de instrumentos e processos complicados de preparação. Além disso traz a vantagem de um bom controle de homogeneidade, tamanho de partícula, composição química e fases morfológicas [22].

Existe um diferença de comportamento dos vários tipos de estrutura do óxido de zinco, devido ao tamanho e aos limites de grão, grãos muito pequenos e suficientes limites de grão são eficientes para o surgimento e aprimoramento de algumas propriedades, como a propriedade fotocatalítica [3]. Esta presença de defeitos faz possível a dopagem do óxido de zinco com outros materiais, o que provoca o controle de morfologia $\mathrm{e}$ consequentemente as propriedades.

O bismuto tem potencial dopante para várias aplicações devido a conversão entre os estados de valência do bismuto ou a existência de muitos estados de valência de bismuto em diferentes hospedeiros [11]. O bismuto tem a habilidade reduzir o band gap nos semicondutores, deslocando a banda de adsorção do semicondutor puro e modificando assim a taxa de separação de portadores de carga induzidos por fotoinduzidos[8].

Por meio deste potencial dopante e dos defeitos encontrados no óxido de zinco, foi realizada a síntese hidrotermal assistida por microondas. Com objetivo de avaliar as concentrações de bismuto no óxido de zinco, e a morfologia estrutural que este método de síntese produz.

\section{Metodologia}

Os materiais utilizados foram nitrato de zinco, nitrato de bismuto, hidróxido de amônio e água Milli-Q [6]. Foram estudadas as concentrações de dopagem 1, 3 e $6 \%$ de bismuto no óxido de zinco.

$\mathrm{O}$ nitrato de zinco foi dissolvido em água Milli-Q à temperatura ambiente e homogeneizado durante 30 minutos 
utilizando um agitador magnético. Após o nitrato de bismuto foi adicionado e homogeneizado novamente durante 30 minutos. Depois adicionou-se o hidróxido de amônio para equilibrar o $\mathrm{pH}$.

A síntese foi realizada em tempos diferentes $(5,10$ e 20 minutos) a $200^{\circ} \mathrm{C}$. Após a reação, o produto foi centrifugado e lavado com água Milli-Q até o seu $\mathrm{pH}$ estabilizar. $\mathrm{O}$ produto foi seco a $100{ }^{\circ} \mathrm{C}$ durante $1 \mathrm{~h}$ e queimado a $400{ }^{\circ} \mathrm{C}$ durante $3 \mathrm{~h}$.

As fases cristalinas foram determinadas por difração de raios $\mathrm{X}$ (XRD) com equipamentos Philips usando radiação de $\mathrm{Cu}$ $\mathrm{K} \alpha(\lambda=1,54184 \mathrm{~A})$ operando a $40 \mathrm{kV}$ e $40 \mathrm{~mA}$ com $2 \theta$ de 10-90 ○. O tamanho médio do cristalito foi calculado pela equação de Scherrer.

As características morfológicas dos óxidos de zinco dopados com bismuto foram avaliadas por microscopia eletrônica de varredura (SEM). A espectroscopia de fotoluminescência foi realizada para caracterizar a presença de defeitos ou alteração óptica com doping [23]. O intervalo de banda foi determinado em um espectrofotômetro Cary 5000, com um comprimento de onda de 200 a $800 \mathrm{~nm}$.

\section{Resultados e discussões}

A Figura 1 apresenta os difratogramas do óxido de zinco e suas diferentes dopagens com bismuto (1, 3 e 6\%). Como visto anteriormente os picos característicos do óxido de zinco (marcados com círculo) podem ser vistos tanto no óxido de zinco sem dopante como nos do óxido de zinco com dopagem.

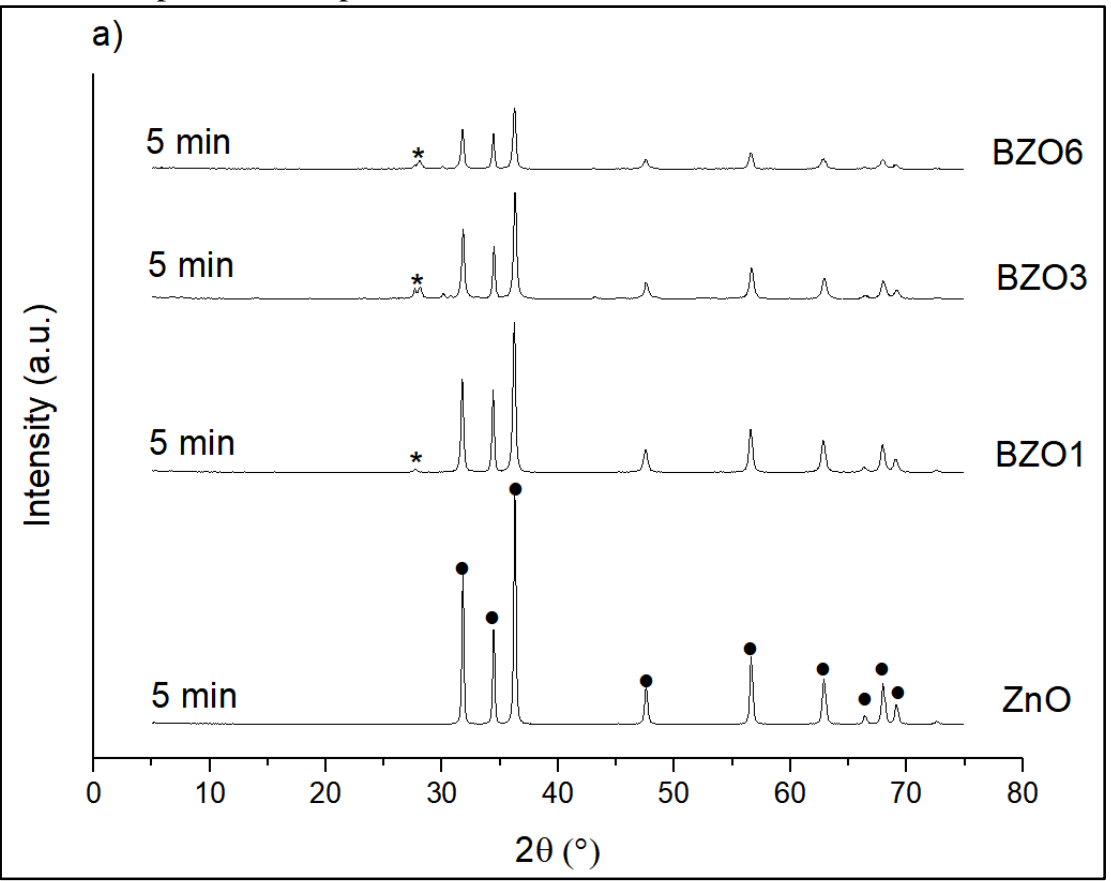

Figura 1 - Difratograma do Zno dopado com bismuto sintetizado por MaHS a $200{ }^{\circ} \mathrm{C}$, por 5 minutos (ZnO, BZO1, BZO3, BZO6).

Entretanto, conforme o aumento da dopagem com bismuto menor a intensidade dos picos característicos do óxido de zinco. A amostra BZO3 que possui 3\% de dopagem de bismuto que obteve os picos mais característicos quanto a dopagem. Quanto ao BZO6, há uma baixa intensidade do pico de bismuto em relação ao mesmo pico em BZO3, isto ocorre devido a diferente localização que os íons de bismuto criaram, porém não foram detectados outros picos de fase de impureza [11].

Os picos adicionais, marcados com um asterisco, aparecem juntamente com a fase do $\mathrm{ZnO}$ para 3 e $6 \%$ em peso de bismuto dopado nas amostras, [6] um pico fraco torna-se mais evidente com o aumento do bismuto que poderia ser atribuído ao óxido de bismuto polimorfo $\left(\mathrm{Bi}_{2} \mathrm{O}_{3}\right)$. Há também a hipótese da presença de um polimorfo segregado com óxido de bismuto [8], já que a presença de uma fase de impurezas secundária é bastante típica e pode causar complicações se tiver uma faixa de banda similar (mas não idêntica) ao composto ternário [24].

A Figura 2 apresenta o difratograma do produto da síntese hidrotermal assistida por microondas do óxido de zinco e seus diferentes tempos (5,10 e 20 minutos) no processo do microondas. Conforme a análise dos resultados confirmou-se o tipo de estrutura wurtzita pela fase hexagonal do óxido de zinco [2, 11, 25-28]. Sendo confirmado, conforme [4], fortes picos de difração aparecendo em $2 \theta=31.8^{\circ}, 34.3^{\circ}$ e $36.5^{\circ}$, correspondendo aos planos (100), (002) e (101).

O tempo do processo de síntese escolhido foi o de 5 minutos, pois conforme o aumento do tempo ocorre a diminuição 
da intensidade dos picos característicos do óxido de zinco. Também foi possível notar maior intensidade do pico característico da dopagem do bismuto, demonstrado pelo asterisco no difratograma.

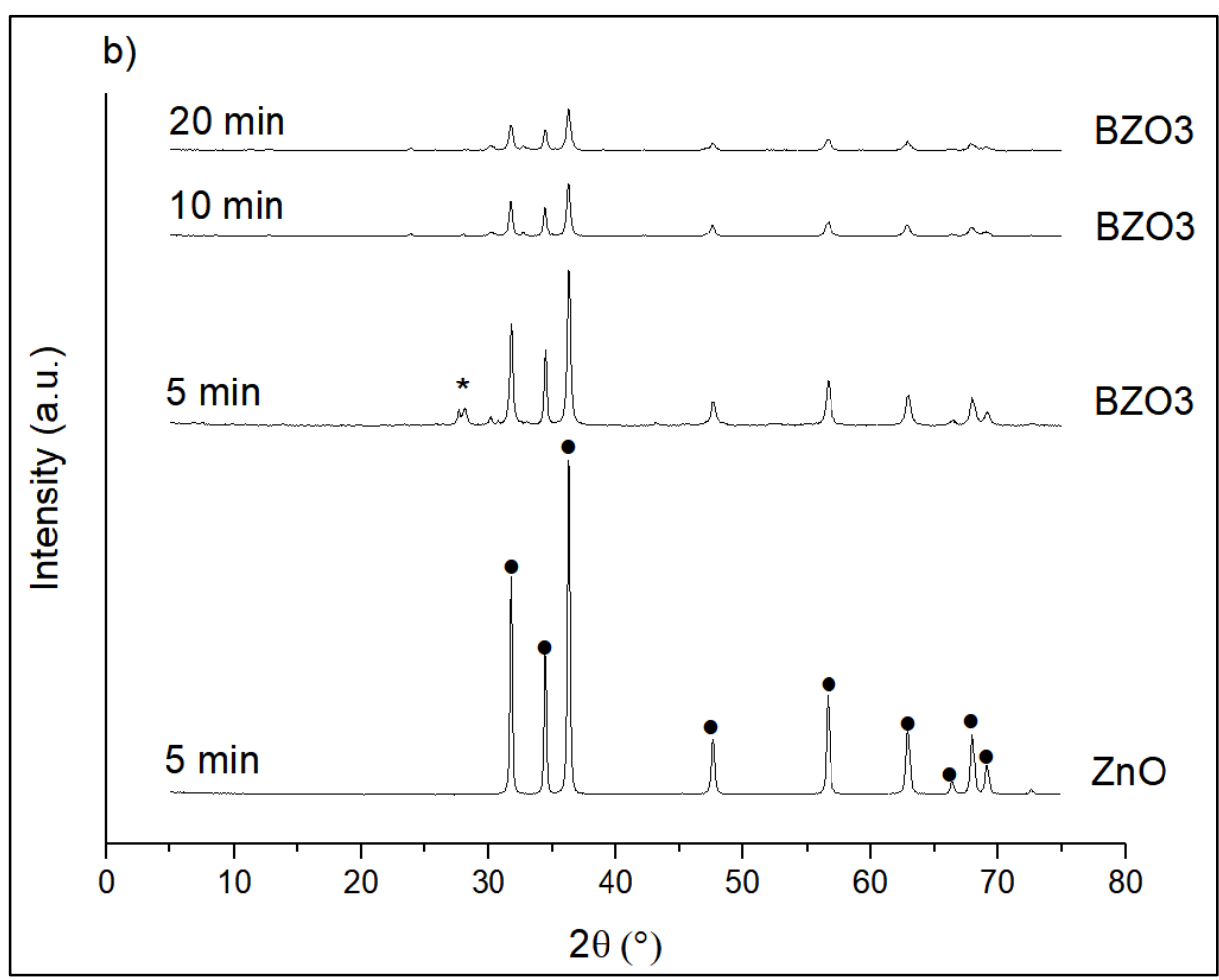

Figura 2 - Difratograma do ZnO dopado com bismuto, sintetizado por MaHS a $200{ }^{\circ} \mathrm{C}$ por 5,10 e 20 minutes (ZnO, BZO1, BZO3, BZO6).

Os tamanhos dos cristalitos pela equação de Scherrer se encontram na Tabela 1, levando em conta o pico de maior intensidade do óxido de zinco. Mesmo com o aumento da dopagem de bismuto no óxido de zinco não há uma interferência quanto ao tamanho do cristalino, todas os teores de dopagem obtiveram o mesmo tamanho de cristalito. Porém com a dopagem ocorre a diminuição do tamanho do cristalito em relação ao óxido de zinco puro. O mesmo acontece com o band gap, os resultados demonstram que o band gap diminui com a dopagem do bismuto, como mostra [6-8, 29]. O band gap pode ser afetado pela morfologia, tamanho de cristalito e área superficial [11].

Tabela 1 - Concentração de dopante, tamanho do cristalito e band gap do óxido de zinco e suas dopagens.

\begin{tabular}{cccc}
\hline Denominação & $\begin{array}{c}\text { Concentração } \\
\text { de dopante }\end{array}$ & $\begin{array}{c}\text { Tamanho } \\
\text { do cristalito }\end{array}$ & $\begin{array}{c}\text { Band gap } \\
(\mathbf{e V})\end{array}$ \\
\hline Zno & $0 \%$ & $8,37 \mathrm{~nm}$ & $3,34 \mathrm{eV}$ \\
BZO1 & $1 \%$ & $6,98 \mathrm{~nm}$ & $2,79 \mathrm{eV}$ \\
BZO3 & $3 \%$ & $6,99 \mathrm{~nm}$ & $3,01 \mathrm{eV}$ \\
BZO6 & $6 \%$ & $6,98 \mathrm{~nm}$ & $2,8 \mathrm{eV}$ \\
\hline
\end{tabular}

Pela utilização do MEV foram analisadas as morfologias das amostras de óxido de zinco e óxido de zinco dopado com bismuto conforme mostra a Figura 3. Nas imagens (a, c, e, g) foi possível notar a formação de aglomerados devido ao tamanho das partículas, isto foi encontrado na literatura $[11,25]$, e que não há mudança de com a dopagem.

O formato denominado flor, como também espículas, pode ser observado em todas amostras $(b, d, f, g)$, tanto no óxido de zinco dopado com bismuto como no óxido de zinco sem dopagem este formato foi encontrado na literatura onde se produz óxido de zinco por síntese hidrotermal assistida por microondas $[9,22]$.

A Figura 4 mostra os espectros do óxido de zinco e óxido dopado com bismuto em diferentes teores. A alta concentração de defeitos intrínsecos e impurezas produz bandas alargadas, e o aumento destes defeitos e impurezas, aumenta a intensidade [28, 30-32]. Este aumento pode ser visto nos espectros, conforme aumenta a dopagem de bismuto, aumenta a intensidade. Uma banda característica foi detectada, entre 420-440 nm, que é considerada a região do espectro de cor vermelha. 


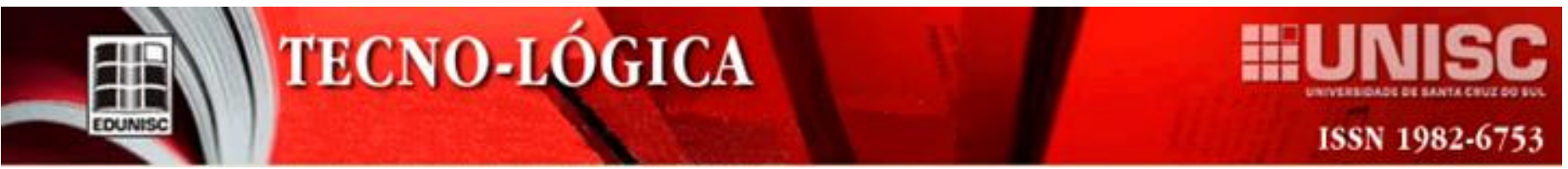
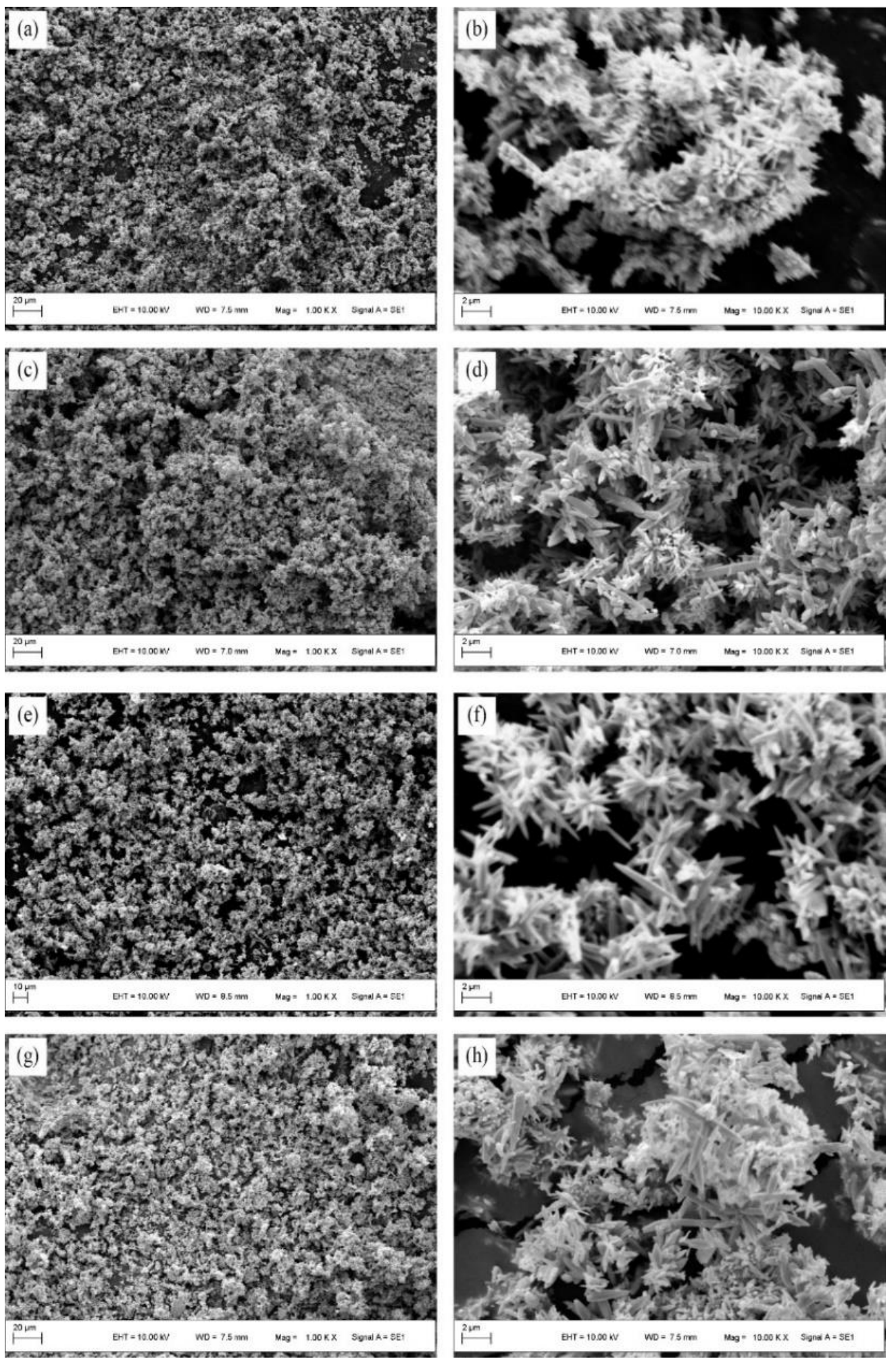

Figura 3 - MEV realizado com aproximação 1000x (a, c, e, g); e com aproximação 10000x (b, d, f, h) do óxido de zinco e suas dopagens (ZnO, BZO1, BZO3, BZO6). 

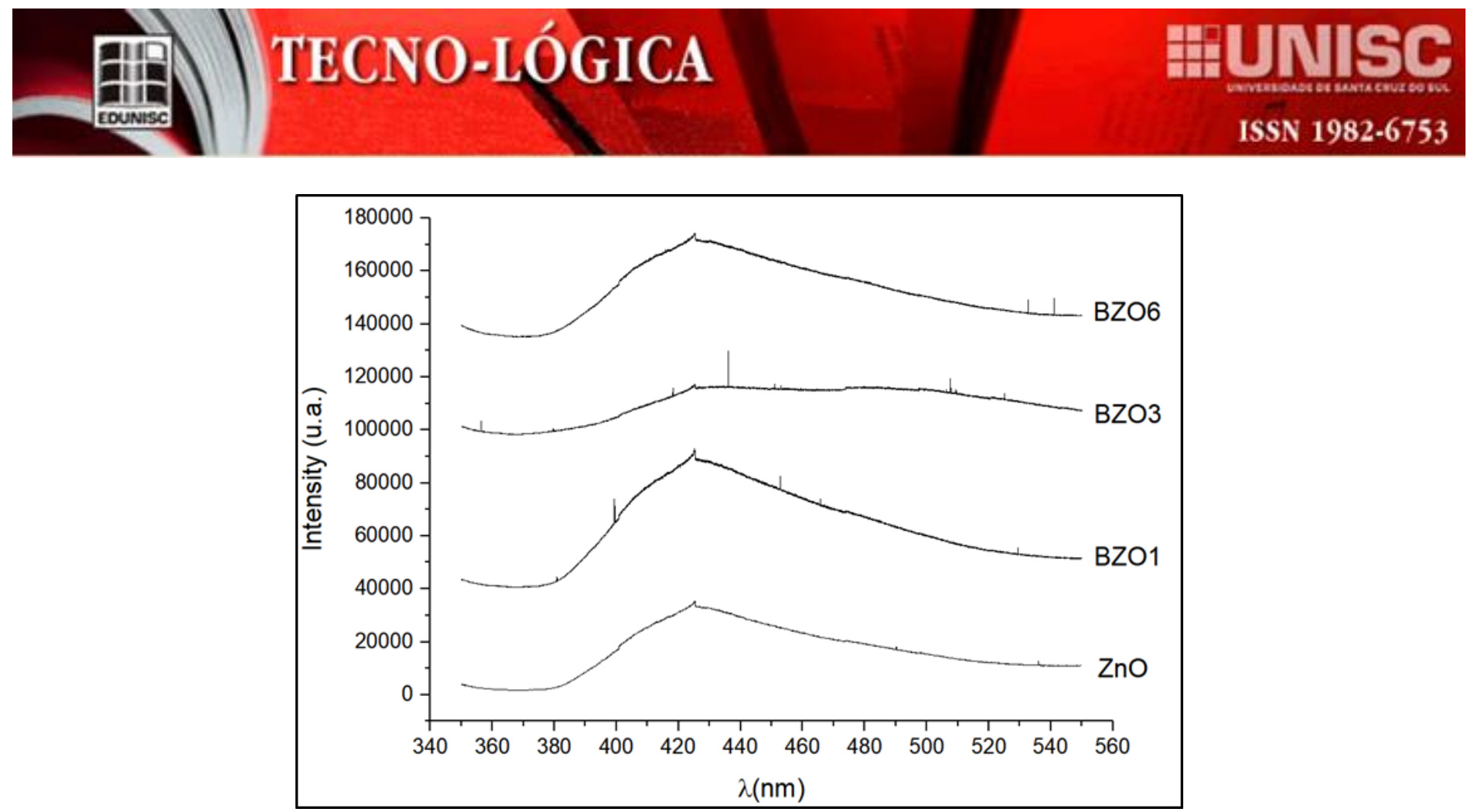

Figura 4 - Espectros de fotoluminescência do óxido de zinco e suas dopagens ( $\mathrm{ZnO}, \mathrm{BZO}$, BZO3, BZO6).

\section{Conclusões}

Sintetizamos o óxido de zinco nanoestruturado dopado com bismuto através de um método de microondas assistido hidrotermal. A introdução do dopante de bismuto demonstra resultados diferentes, dependendo da via de síntese adotada [8].

O melhor tempo de síntese foi de 5 minutos a $200^{\circ} \mathrm{C}$. A morfologia produzida é denominada flor, tanto para o $\mathrm{ZnO}$ como para o óxido de zinco dopado com bismuto. O tamanho do cristalito variou de $5,82 \mathrm{~nm}$ a $8,37 \mathrm{~nm}$ da equação de Scherrer. O intervalo de band gap para as diferentes concentrações de dopantes foi 2,79-3,34 eV indicando seu uso potencial em diferentes aplicações envolvendo fotodegradação.

\section{SYNTHESIS OF NANOSTRUCTURED ZINC OXIDE DOPED WITH BISMUTH BY A MICROWAVE- ASSISTED HYDROTHERMAL METHOD AND THEIR CHARACTERIZATION}

ABSTRACT: Zinc oxide due to the presence of intrinsic defects makes it possible to doping with other materials, such as bismuth. The BZO (zinc oxide doped with bismuth) prepared via microwave-assisted hydrothermal synthesis (MaHS) was studied for its dopant percentage and morphological characterization. The products were characterized via XRD, SEM, and PL; band gap calculations by transmittance spectroscopy. The results indicate that BZO nanostructures were obtained with crystallite sizes ranging from 5,82 to $8,37 \mathrm{~nm}$. The same flower like structure was formed with diferents dopants. PL demonstrates that defects increase with bismuth doping. The band-gap of $2,79-3.3 \mathrm{eV}$ has potential use in photodegradation applications.

Keywords: Hydrothermal synthesis. Microwave. Zinc oxide. Dopant. Bismuth.

\section{Referências}

1. SAMADI, M., et al., Recent progress on doped $\mathrm{ZnO}$ nanostructures for visible-light photocatalysis. Thin Solid Films, 2016. 605(Supplement C): p. 2-19.

2. LEE, K.M., et al., Recent developments of zinc oxide based photocatalyst in water treatment technology: A review. Water Research, 2016. 88(Supplement C): p. 428-448.

3. CAO, M., et al., Shape-controlled synthesis of flower-like $\mathrm{ZnO}$ microstructures and their enhanced photocatalytic properties. Materials Letters, 2017. 192(Supplement C): p. 1-4.

4. BARRETO, G., et al., Microwave Assisted Synthesis of $\mathrm{ZnO}$ Tridimensional Nanostructures. Procedia Materials Science, 2015. 8(Supplement C): p. 535-540.

5. LEITNER, J., et al., Thermodynamic properties of nanostructured $\mathrm{ZnO}$. Applied Materials Today, 2018. 10(Supplement C): p. 1-11.

6. PRAKASH, T., et al., Structural, morphological and optical properties of Bi-doped $\mathrm{ZnO}$ nanoparticles synthesized by a microwave irradiation method. Journal of Materials Science: Materials in Electronics, 2015. 26(7): p. 4913-4921.

7. $\quad$ ŠTENGL, V., et al., $\mathrm{ZnO} / \mathrm{Bi} 2 \mathrm{O} 3$ nanowire composites as a new family of photocatalysts. Powder Technology, 2015. 270(Part A): p. 83-91.

8. MOSTONI, S., et al., Tailored routes for home-made Bi-doped $\mathrm{ZnO}$ nanoparticles. Photocatalytic performances towards o-toluidine, a toxic water pollutant. Journal of Photochemistry and Photobiology A: Chemistry, 2017. 332(Supplement C): p. 534-545. 
9. HASANPOOR, M., M. ALIOFKHAZRAEI, AND H. DELAVARI, Microwave-assisted Synthesis of Zinc Oxide Nanoparticles. Procedia Materials Science, 2015. 11(Supplement C): p. 320-325.

10. ZHONG, J.B., et al., Fabrication of Bi3+-doped $\mathrm{ZnO}$ with enhanced photocatalytic performance. Applied Surface Science, 2012. 258(11): p. 4929-4933.

11. PATHAK, T.K., H.C. SWART, AND R.E. KROON, Influence of Bi doping on the structure and photoluminescence of $\mathrm{ZnO}$ phosphor synthesized by the combustion method. Spectrochimica Acta Part A: Molecular and Biomolecular Spectroscopy, 2018. 190(Supplement C): p. 164-171.

12. DIKICI, T., Temperature-dependent growth of $\mathrm{ZnO}$ structures by thermal oxidation of $\mathrm{Zn}$ coatings electrodeposited on steel substrates and their photocatalytic activities. Ceramics International, 2017. 43(11): p. 82898293.

13. ONG, C.B., L.Y. NG, AND A.W. MOHAMMAD, A review of ZnO nanoparticles as solar photocatalysts: Synthesis, mechanisms and applications. Renewable and Sustainable Energy Reviews, 2018. 81(Part 1): p. 536-551.

14. DAS, S. AND V.C. SRIVASTAVA, Synthesis and characterization of $\mathrm{ZnO} / \mathrm{CuO}$ nanocomposite by electrochemical method. Materials Science in Semiconductor Processing, 2017. 57: p. 173-177.

15. CALVET, I., et al., Synthesis, deposition and crystal growth of CZTS nanoparticles onto ceramic tiles. Boletín de la Sociedad Española de Cerámica y Vidrio, 2015. 54(5): p. 175-180.

16. RAYATHULHAN, R., B.K. Sodipo, and A.A. Aziz, Nucleation and growth of zinc oxide nanorods directly on metal wire by sonochemical method. Ultrasonics Sonochemistry, 2017. 35: p. 270-275.

17. YE, Z., et al., Na-doped $\mathrm{ZnO}$ nanorods fabricated by chemical vapor deposition and their optoelectrical properties. Journal of Alloys and Compounds, 2017. 690: p. 189-194.

18. JIA, W., et al., Synthesis of Al-ZnO nanocomposite and its potential application in photocatalysis and electrochemistry. Inorganic Chemistry Communications, 2018. 88: p. 51-55.

19. ZGURA, I., et al., Wet chemical synthesis of $\mathrm{ZnO}-\mathrm{CdS}$ composites and their photocatalytic activity. Materials Research Bulletin, 2018. 99: p. 174181

20. WANG, Y., et al., Synthesis of $\mathrm{ZnO}$ nanoparticles from microemulsions in a flow type microreactor. Chemical Engineering Journal, 2014. 235: p. 191-197.

21. MUKHAMEDSHINA, D., et al., Fabrication and study of sol-gel ZnO films for use in Si-based heterojunction photovoltaic devices. Modern Electronic Materials, 2017.

22. MIRZAEI, A. and G. NERI, Microwave-assisted synthesis of metal oxide nanostructures for gas sensing application: A review. Sensors and Actuators B: Chemical, 2016. 237(Supplement C): p. 749-775.

23. TAWALE, J.S., et al., Microstructural evolution and photoluminescence performanance of nickel and chromium doped $\mathrm{ZnO}$ nanostructures. Materials Chemistry and Physics, 2018. 205: p. 9-15.

24. ROY, D., et al., On the measured optical bandgap values of inorganic oxide semiconductors for solar fuels generation. Catalysis Today, 2018. 300(Supplement C): p. 136-144.

25. BUSTOS-TORRES, K.A., et al., Influence of the morphology of $\mathrm{ZnO}$ nanomaterials on photooxidation of polypropylene/ $\mathrm{ZnO}$ composites. Materials Science in Semiconductor Processing, 2017. 68(Supplement C): p. $217-225$

26. GUAN, W., et al., Theoretical and experimental investigations of the thermoelectric properties of Al-, Bi- and Sn-doped $\mathrm{ZnO}$. Materials Science in Semiconductor Processing, 2017. 66(Supplement C): p. 247-252.
27. HEZAM, A., et al., Synthesis of heterostructured $\mathrm{Bi} 2 \mathrm{O} 3-\mathrm{CeO} 2-\mathrm{ZnO}$ photocatalyst with enhanced sunlight photocatalytic activity. Ceramics International, 2017. 43(6): p. 5292-5301.

28. UDOM, I., et al., One dimensional-ZnO nanostructures: Synthesis, properties and environmental applications. Materials Science in Semiconductor Processing, 2013. 16(6): p. 2070-2083.

29. KIRANKUMAR, V.S. and S. Sumathi, Catalytic activity of bismuth doped zinc aluminate nanoparticles towards environmental remediation. Materials Research Bulletin, 2017. 93(Supplement C): p. 74-82.

30. LV, Y., et al., Growth mechanism and photoluminescence property of hydrothermal oriented $\mathrm{ZnO}$ nanostructures evolving from nanorods to nanoplates. Journal of Alloys and Compounds, 2017. 718: p. 161-169.

31. YI, X.Y., et al., Structural, morphological, photoluminescence and photocatalytic properties of Gd-doped ZnO films. Thin Solid Films, 2017. 636: p. 339-345.

32. SOUSA, R.B., et al., Síntese, caracterização e propriedade fotoluminescente de tungstato de bário puro e dopado com hólmio. Cerâmica, 2015. 61(358): p. 224-235. 